\title{
On the diffusion law of additional stress and bearing capacity of interbed
}

\section{foundation in Northern Anhui}

\author{
Xiu-he Chen ${ }^{1}$, Ze-cheng $\mathrm{Chi}^{2}$, Shan-xiong Chen ${ }^{2}$, Fei YU \\ 1Anhui Communications Planning, Design and Research Institute, Hefei 230041, China. \\ 2 Institute of Rock and Soil Mechanics, Chinese Academy of Sciences, Wuhan 430071, China; \\ yufei8720@163.com
}

Keywords: interbed foundation; bearing capacity; additional stress; diffusion law.

Abstract: The shortage of foundation bearing capacity would pose damage to miniature structures on highway, threatening the security of highway. Layered foundations are widely distributed in Northern Anhui, the traditional methods of computing the bearing capacity and additional stress disagree with the situation of complexity and uncertainty multi-layered foundation. To study the characteristics of additional stress and bearing capacity of multi-layered foundation in Northern Anhui using numerical method of FLAC3D and analyze the influencing factors of soil structure, soil thickness and basis width on the diffusion laws and bearing capacity. The results can be references in the site survey and engineering design of multi-layered foundation.

\section{Introduction}

There are lots of miniature structures of highway in Northern Anhui. Geological conditions of the region is complex, so the bridge detection and evaluation of the highway is one of technical problems.

Most of the earth's surface in Northern Anhui is covered by debris formed by The Yellow River and the huaihe river flood.Geological conditions have the characteristics of complexity and uncertaint $y^{[1-3]}$, including:1) The characteristic of layered sedimentary is significant:The thickness of layer is thin, containing a great many soil thickness; 2) There is a number of calcareous concretion soil: most of calcareous concretion in the clay and silty clay located in the surface of soil, so the soil is not uniform; 3)Newly sedimentary silty soil is widely distributed around the region, so bearing capacity has a big change. In a word, reasonable testing and evaluation of foundation bearing capacity in Northern Anhui can ensure the safety of the construction and operation.

Small bridge culverts of highway in Northern Anhui are widely distributed around the region, therefore detailed reconnaissance is impossible in the early. The traditional methods, for example, look-up table method and In situ tests, is difficult to apply ${ }^{[4]}$.The main reason: the diffusion law of additional stress of multi-layered foundation is complex. Foundation bearing capacity influenced by lots of factors is difficult to sure $\mathrm{e}^{[5-8]}$.

To study the characteristics of additional stress and bearing capacity of multi-layered foundation in Northern Anhui using numerical method of FLAC3D and analyze the influencing factors of soil structure, soil thickness and basis width on the diffusion laws and bearing capacity. The results can be references in the site survey and engineering design of multi-layered foundation. 


\section{The Program of Calculation and Analysis in Multi-layered Foundation}

Calculation Model. According to the characters of geological structure of multi-layered foundation, FLAC3D is adopted to establish the geological generalized model. The reality is a semi-infinite space's axisymmetric problem, so establishing the axisymmetric model whose symmetry plane is the foundation center. Miniature structures general use shallow foundation which is limited in load impact depth,hence the model pay attention to the soil influenced by load.The foundation soil will be divided into four layers is same from the actual situation. The thickness of every layer is about 1-2 meters. The scope of load is same from actual situation.The foundation depth is 20 meters. Limiting the horizontal displacement of model on both sides of the boundary and the vertical displacement on the bottom boundary.

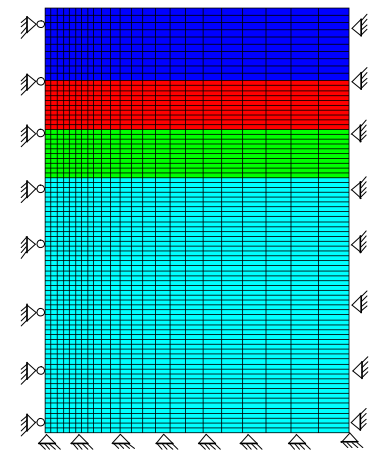

Figure 1theanalysis calculation grid of the model

Calculation Parameters.In order to know the diffusion law of additional stress of multi-layered foundation,the foundation soil is divided into soft and hard soil in the model. Calculation parameters are determined by experiment and calculation, as shown in table 1 :

Table 1 calculation parameters

\begin{tabular}{|c|c|c|c|c|}
\hline \multicolumn{1}{|c|}{} & & & & \\
\hline Type & modulus of deformation/MPa \\
Parameter & & Poisson's ratio & Cohesive force/kPa & Angle of Internal Friction/ \\
\hline Soft soil & 6 & & & \\
\hline Hard soil & 20 & 0.4 & 6 & 10 \\
\hline
\end{tabular}

Analysis Case.In order to analyze additional stress and bearing capacity of multi-layered foundation's law influenced by soil structure,each layer thickness, width, Multiple analysis condition is designed, as shown in table 2. Among them, the conditions of 25-27 are soft soil foundation, but in the surface of soil foundation have hard soil with different thickness. The conditions of 28-30 are hard soil foundation, but the upper hard crust lying with different thickness. The conditions of 31-36 analyze change law when the load's width is different in the foundation with weak intercalated layer. 
Table2working condition of calculation

\begin{tabular}{|c|c|c|c|c|c|c|c|}
\hline \multirow{2}{*}{$\begin{array}{c}\text { Condition } \\
\text { number }\end{array}$} & \multirow{2}{*}{ the width of foundation/m } & \multicolumn{2}{|c|}{$\begin{array}{c}\text { The first layer of } \\
\text { basement }\end{array}$} & $\begin{array}{c}\text { The second layer of } \\
\text { basement }\end{array}$ & \multicolumn{2}{c|}{$\begin{array}{c}\text { The third layer of } \\
\text { basement }\end{array}$} \\
\cline { 3 - 8 } & & $\begin{array}{c}\text { Thickness } \\
\text { m }\end{array}$ & type & $\begin{array}{c}\text { Thickness } \\
/ \mathrm{m}\end{array}$ & type & $\begin{array}{c}\text { Thickness } \\
/ \mathrm{m}\end{array}$ & type \\
\hline 25 & 4.0 & 2.0 & Soft & 1.0 & Hard & 2.0 & Soft \\
\hline 26 & 4.0 & 2.0 & Soft & 2.0 & Hard & 2.0 & Soft \\
\hline 27 & 4.0 & 2.0 & Soft & 3.0 & Hard & 2.0 & Soft \\
\hline 28 & 4.0 & 1.0 & Soft & 1.0 & Hard & 2.0 & Soft \\
\hline 29 & 4.0 & 1.5 & Hard & 1.0 & Soft & 2.0 & Hard \\
\hline 30 & 4.0 & 2.5 & Hard & 1.0 & Soft & 2.0 & Hard \\
\hline 31 & 1.5 & 2.0 & Hard & 1.0 & Soft & 2.0 & Hard \\
\hline 32 & 2.0 & 2.0 & Hard & 1.0 & Soft & 2.0 & Hard \\
\hline 33 & 2.5 & 2.0 & Hard & 1.0 & Soft & 2.0 & Hard \\
\hline 34 & 3.0 & 2.0 & Hard & 1.0 & Soft & 2.0 & Hard \\
\hline 35 & 3.5 & 2.0 & Hard & 1.0 & Soft & 2.0 & Hard \\
\hline 36 & 5.5 & 2.0 & Hard & 1.0 & Soft & 2.0 & Hard \\
\hline
\end{tabular}

\section{The Diffusion Law of Additional Stress of Interbed Foundation}

Although conditions are different, the diffusion law of additional stress of interbed foundation is consistent and obvious.Usingthe conditions of 26 to explain this.

The Diffusion Law of Additional Stress of Foundation. The change of additional stress of foundation can be divided into the following stages:

1) The Stage of Proportional Limit. In this stage,additional stress first began to expand under foundation angular point, and spread to the deep of foundation. At this time the additional stress is mainly distributed in the upper hard soil layer and additional stress of deep soil is small.Most of weak intercalated layer is damaged, so the soft soil cannot bear more load.

2) The Stage of Proportional Limit to Plastic Load. In this stage, although most of weak intercalated layer is damaged, the lower hard soil can still bear load, additional stress began to expand in it. The scope of additional stress passed by upper soft soil is still large, though the bearing of deep hard soil is small. As shown in figure 2, cloud map of additional stress of foundation in Load of $320 \mathrm{kpa}$.

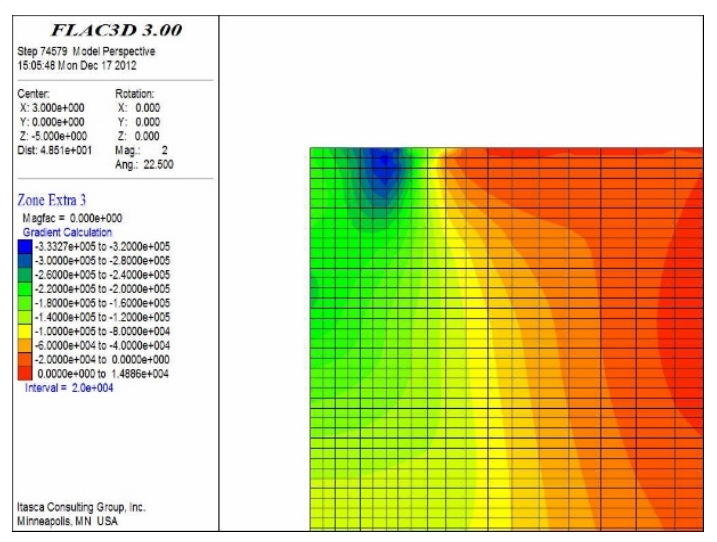

Figure 2cloud map of additional stress of foundation in Load of $320 \mathrm{kpa}$ 
3) Limit State. In limit state, additional stress still expand to deep soil. The law is same with before.

The Change of Level's Additional Stress of Bottom of Foundation in Different Cases.According to the analysis cases, researching the change of level's additional stress 3 meters under the bottom of foundation from width of weak intercalated layer, width of hard crust lying and width of foundation.

It can be seen from figure 3: In upper hard crust lying,additional stress appears stress concentration phenomenon in horizontal direction. The biggest of additional stress locates under the boundary of base. On the border of the soft and hard soil, stress concentration phenomenon disappeared under the border.

It can be seen from figure 4: The width of weak intercalated layer is small, with the increase of thickness of hard crust lying, additional stress turns bigger in horizontal direction.

It can be seen from figure 5: In hard crust lying, additional stress appears stress concentration phenomenon in horizontal direction. The biggest of additional stress locates under the boundary of base.On the border of the soft and hard soil, stress concentration phenomenon disappeared. With the increase of width of foundation, additional stress turns bigger. It shows the scope of soil bearing load changes big.

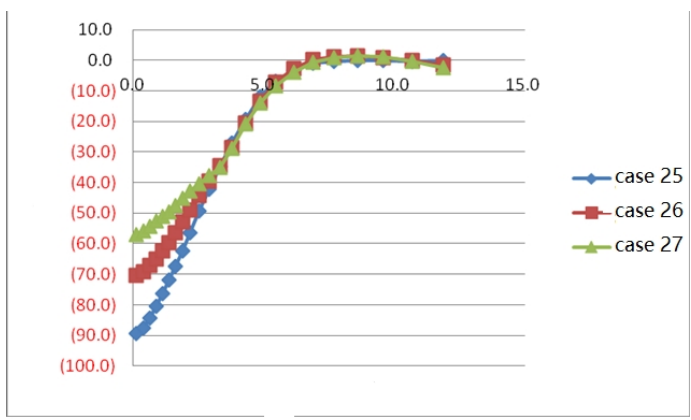

Figure 3 comparing of additional stress in different width of weak intercalated layer

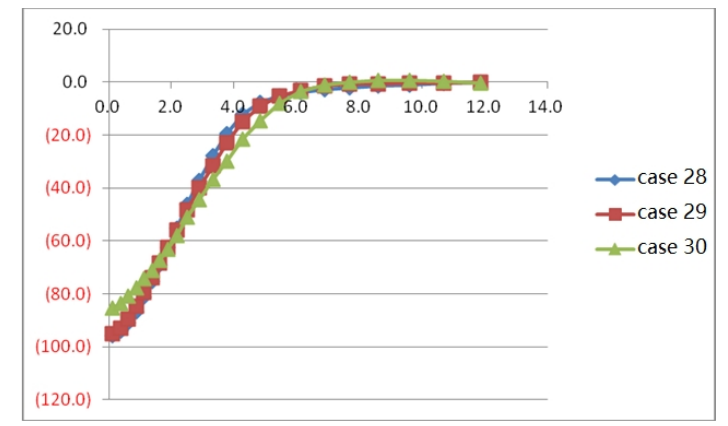

Figure 4 comparing of additional stress in different width of hard crust lying

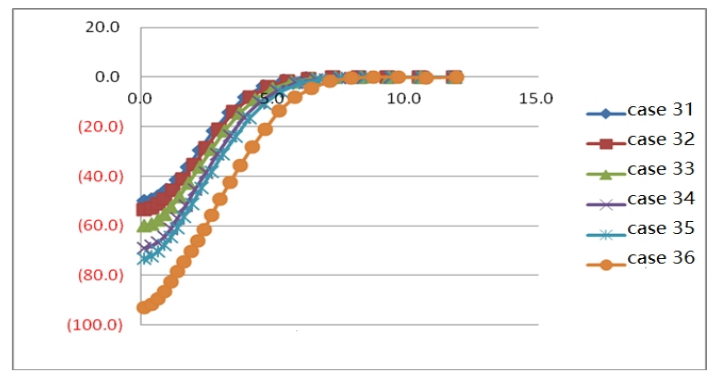

Figure 5 comparing of additional stress in different width of basement 
The Diffusion Scope of Additional Stress in Different Cases.According to the analysis cases, studying the diffusion scope of additional stress from width of weak intercalated layer, width of hard crust lying and width of foundation.

Calculating shows: In cases with different width of weak intercalated layer, the diffusion Angle of additional stress of multi-layered foundation is basically same. In addition, with the decrease of soft soil's width, effect's depth of additional stress turns bigger.

In cases with different width of upper hard crust lying, width of weak intercalated layer is same, so effect's depth is same too. With the increase of upper hard crust lying's width, the effect's scope of additional stress turns bigger in horizontal direction.

In cases with different width of base,additional stress spread at about $45^{\circ}$ angle. When the diffusion curve reaches the border of the soft and hard soil,with the increacing of depth, the curve of additional stress declined. With the increase of base's width, the effect's depth of additional stress turns big.

\section{The Diffusion Law and Bearing Capacity of Multi-layered Foundation}

The Bearing Capacity of Multi-layered Foundation.In this section, researching the bearing capacity of multi-layered foundation and its influencing factors, evaluting its influencing scope.Making the bearing capacity of multi-layered foundation be tested and evaluted more quickly and reasonably.

The calculation results show: P - S curve can be obviously divided into three stages, when soil containing weak intercalated layer. It has obvious the proportional limit and the plastic load. The reason is the width of weak intercalated layer is small, the whole ground occurred shear failure under the influence of ultimate load.

The Ultimate Bearing Capacity ofMulti-layered Foundation and Its Change Rule.According to the calculation results, we can kown: calculating the bearing capacity of foundation by using two P-S curves have not obvious inflection point. Using the first method to make sure the case has obvious inflection point. The calculation results of multi-layered foundation are all in firgure 6 .

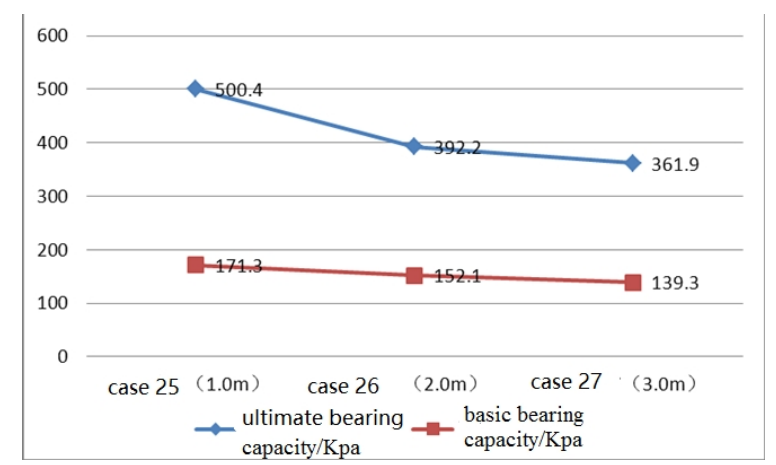

Figure 6 comparing of ultimate bearing capacity and characteristic value in different width of weak intercalated layer

It can be seen from figure 6: with the increase of width of weak intercalated layer, the ultimate bearing capacity change small. The reason is weak soil intensity is small and occured lateral plastic damage, so the stress diappeared qucikly in soft soil, and with the increacing of soil layer's width, the ultimate bearing capacity declined.

1) The Change of Bearing Capacity in Different Width of Upper Hard Crust Lying. It can be seen from figure 7: under the condition of different width of the first hard crust lying, 
with the increacing of its width, the ultimate bearing capacity rising, because hard soil intensity is large and can bear considerable load.

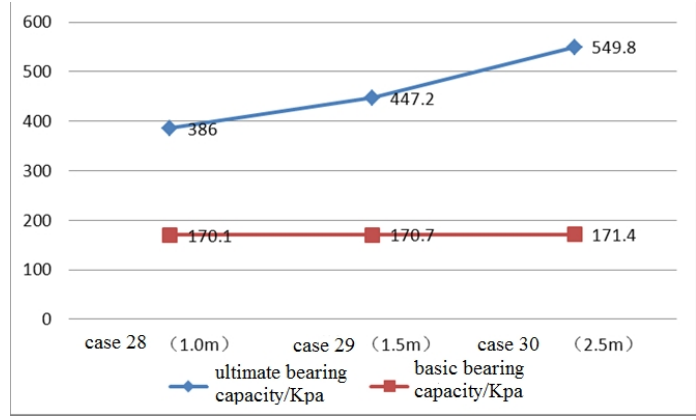

Figure 7 comparing of ultimate bearing capacity and characteristic value in different width of hard soil

2) The Change of Bearing Capacity in Different Width of Base. It can be seen from figure 8: under certain conditions, with the increasing of foundation's width, the ultimate bearing capacity and characteristic value of bearing capacity rising. This reason is the width of weak intercalated layer is small, most of bearing capacity is controlled by hard soil, the foundation in the case is similar to homogeneous foundation.

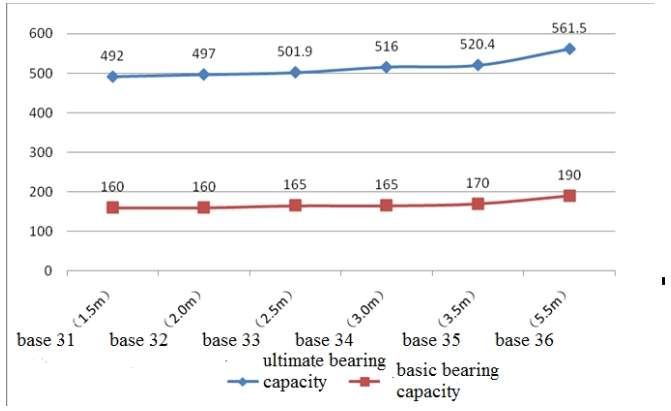

Figure 8 comparing of ultimate bearing capacity and characteristic value in different width of basement

The Analysis and Evaluation of Influence Factors whose Characteristic Value of Bearing Capacity.In this section, researching the bearing capacity of multi-layered foundation and its influencing factors, evaluting its influencing scope. Making the bearing capacity of multi-layered foundation be tested and evaluted more quickly and reasonably.

1) The Change of Characteristic Value of Bearing Capacity in Different Conditions of Base. It can be seen from figure 5 and 6: compared with homogeneous hard soil foundation, bearing capacity of multi-layered foundation has the following rules:

When the hard soil foundation containing soft soil, bearing capacity reduced quickly. When the width of soft soil is about 1 to 3 meters, characteristic value of bearing capacity would decline $29 \%$ to $42 \%$. With the increasing of soft soil layer's width, the characteristic value declined gradually. This shows the width of soft soil has big influence in the characteristic value, we should investigate the ground in practice.

Compared with homogeneous hard soil, with the increasing of hard crust lying's width, the characteristic value of multi-layered foundation declined about $30 \%$. When the thickness of upper hard soil more than 1.5 meters, its width hasn't influence in the characteristic value. The reason is the thickness of upper hard soil hasn't also influence in the scope of characteristic value.

2) The Influence of Base's Width to The Characteristic Value of Bearing Capacity. It can be seen from figure 7: compared with the bearing capacity of the reference, bearing capacity of multi-layered foundation has the following rules: 
With the increasing of base's width, the characteristic value rose slightly by $2 \%$ to $4 \%$ for multi-layered foundation containing soft soil layered.It's different with the result of double-layered foundation, because the width of soft soil layered is samll and the intensity of it is low, transfer effect is small.

\section{Conclusion}

To study the characteristics of additional stress and bearing capacity of multi-layered foundation in Northern Anhui using numerical method of FLAC3D and analyze the influencing factors of soil structure, soil thickness and basis width on the diffusion laws and bearing capacity. The results can be references in the site survey and engineering design of multi-layered foundation.

1)The width of weak intercalated layer and base have influence in bearing capacity of multi-layered foundation. With the increasing of weak intercalated layer's width and bases', the bearing capacity declined.

2)When the width of hard crust lying is not more than 2 meters, it dosen't appear stress concentration phenomenon in horizontal direction.Otherwise, it appears. On the border of the soft and hard soil, stress concentration phenomenon disappeared. Suggest to increase survey point near hard crust lying.

3)For multi-playered foundation containing weak intercalated layer, when additional stress spread to the border of the soft and hard soil,with the increacing of depth, the curve of additional stress declined.Suggest to survey to the maximum depth influenced by additional stress in the center of base.

4) Weak intercalated layer can significantly reduce the ultimate bearing capacity and the characteristic value of foundation. With the increasing of it's width, the bearing capacity declined. Compared with homogeneous hard soil foundation,ultimate bearing capacity and characteristic value of multi-layered foundation containing soft soil layered decreased by approximately $70 \%$ and $40 \%$ respectively.

\section{Reference}

[1]JinQuan. Anhui huaibei plain quaternary system [M]. Beijing: geological publishing house, 1990.

[2]Duan Haipeng, Wang Yaoming. Powder in huaibei area were classified into the bearing capacity of soil foundation research - in Montreal to bengbu highway,forexample [J]. Journal of geological hazards and environmental protection, 2003, 14 (4) : 37-39.

[3] Shan Hongxian, Chen Yong, Liu Zhengyin. such as modern Yellow River delta silty soils to determine bearing capacity $[\mathrm{J}]$. Journal of rock mechanics and engineering, 2006, 25 (S2) : 4089-4096.

[4] Sun Xiang, Liu Xinrong, Zhang Yongxing. Under the hard soft on double-layered foundation finite element analysis [J]. Journal of chongqing university of architecture, 2004, 26 (4) : 41-44.

[5] Zhang Qiyi, Luan Maotian. Composite load cases double-layered foundation ultimate bearing capacity study [J]. Rock and soil mechanics, 2009, 30 (4) : 1131-1136.

[6] D. K. Karamitros, G. D. Bouckovalas, Y. K. Chaloulos and K. I. Andrianopoulos. Numerical analysis of liquefaction-induced bearing capacity degradation of shallow foundations on a two-layered soil profile[J]. Soil Dynamics and Earthquake Engineering, 2013, 44 90-101. 
[7] Yu Yanan, Yu Fei, Dai Zhangjun. Typical geological formation rule and huaibei plain and impact analysis [J]. Science, technology and engineering, 2012, 12 (5) : 1001-1006.

[8] Zhang Qiyi, Wang Qinghua, Wang Qi. On hard under the soft strip footing on two-layer foundation puncture depth study [J]. Journal of railway engineering, 2011, (11) : 36 to 39. 